\title{
Nature of the insulating phases in the half-filled ionic Hubbard model
}

\author{
A. P. Kampf ${ }^{a}$, M. Sekania ${ }^{a}$, G. I. Japaridze ${ }^{a, b}$, and Ph. Brune ${ }^{a}$ \\ ${ }^{a}$ Institut für Physik, Theoretische Physik III, Elektronische Korrelationen und Magnetismus, \\ Universität Augsburg, 86135 Augsburg, Germany \\ ${ }^{b}$ Institute of Physics, Georgian Academy of Sciences, Tamarashvili Str. 6, 380077, Tbilisi, Georgia
}

We investigate the ground-state phase diagram of the one-dimensional "ionic" Hubbard model with an alternating periodic potential at half-filling by numerical diagonalization of finite systems with the Lanczos and density matrix renormalization group (DMRG) methods. We identify an insulatorinsulator phase transition from a band to a correlated insulator with simultaneous charge and bondcharge order. The transition point is characterized by the vanishing of the optical excitation gap while simultaneously the charge and spin gaps remain finite and equal. Indications for a possible second transition into a Mott-insulator phase are discussed.

PACS numbers: 71.10.-w, 71.10.Fd, 71.10.Hf, 71.27.+a, 71.30.+h

\section{INTRODUCTION}

For more than two decades the correlation induced metal-insulator transition and its characteristics has been one of the challenging problems in condensed matter physics [1]. This metal-insulator transition is often accompanied by a symmetry breaking and the development of long range order [2]. In one dimension this ordering can only be related to the breaking of a discrete symmetry. Examples include commensurate charge density waves (CDWs) and Peierls dimerization (bond-order wave, $\mathrm{BOW}$ ) phenomena. In contrast, the transition into the Mott insulating (MI) phase in one dimension is not connected with the breaking of a discrete symmetry [3]. In the MI phase the gapped charge degrees of freedom are uniformly distributed in the system, while the gapless spin degrees of freedom are described by an effective $S=1 / 2$ Heisenberg chain [4].

Due to the different symmetries of the CDW, BOW, and MI phases it is natural to expect that these phases are mutually exclusive. The extended Hubbard model at half-filling with an on-site $(U)$ and a nearest neighbor $(V)$ Coulomb repulsion provides a prominent example with a transition from a MI to a CDW insulator in the vicinity of the $U=2 \mathrm{~V}$ line in the phase diagram [5]. Remarkably, the transition at weak coupling may involve an intermediate BOW phase [6,7]. A similar phase-diagram structure was recently also discovered for the Holstein Hubbard model [8]. The tendency towards BOW order is even more profound for the Hubbard model with an explicit bond-charge coupling where the CDW and MI phases are often separated by a long range ordered Peierls dimerized phase [9].

In recent years particular attention has been given to another example for an extension of the Hubbard model which includes a staggered potential term [10-18]. The corresponding Hamiltonian has been named the "ionic Hubbard model" (IHM); in one dimension it is given by

$$
H=-t \sum_{i, \sigma}\left(1+(-1)^{i} \delta\right)\left(c_{i \sigma}^{\dagger} c_{i+1 \sigma}+H . c .\right)
$$

$$
+U \sum_{i} n_{i \uparrow} n_{i \downarrow}+\frac{\Delta}{2} \sum_{i, \sigma}(-1)^{i} n_{i \sigma}
$$

where $c_{i \sigma}^{\dagger}$ creates an electron on site $i$ with spin $\sigma$ and $n_{i \sigma}=c_{i \sigma}^{\dagger} c_{i \sigma} . \Delta$ is the potential energy difference between neighboring sites, and $\delta$ a Peierls modulation of the hopping amplitude $t$. In the limit $\Delta=\delta=0$, Eq. (1) reduces to the ordinary Hubbard model, the limit $\Delta=0$ and $\delta>0$ is called the Peierls-Hubbard model, and the limit $\Delta>0$ and $\delta=0$ is usually referred to as the IHM. In the following, we will focus mainly on the effect of the on-site modulation $\Delta$, so we implicitly assume $\delta=0$ except where stated otherwise.

The IHM was first proposed and discussed almost 20 years ago in the context of organic mixed-stack chargetransfer crystals with alternating donor $(D)$ and acceptor $(A)$ molecules $\left(\ldots D^{+\rho} A^{-\rho} \ldots\right)[19,10]$. These stacks form quasi-1D insulating chains, and at room temperature and ambient pressure are either mostly ionic $(\rho \approx 1)$ or mostly neutral $(\rho \approx 0)$ [19]. However, several systems undergo a reversible neutral to ionic phase transition i.e. a discontinuous jump in the ionicity $\rho$ upon changing temperature or pressure [20]. Later the IHM has been used in a similar context to describe the ferroelectric transition in perovskite materials such as $\mathrm{BaTiO}_{3}$ $[21,22]$ or $\mathrm{KNbO}_{3}$ [23].

The very presence of at least on transition in the ground state phase diagram of the half-filled IHM model is easily traced by starting from the atomic limit $[24,13]$. For $t=0$, it is obvious that at $U<\Delta$ the ground state of the IHM has two electrons on the odd sites, and no electrons on the even sites corresponding to CDW order with maximum amplitude. On the other hand, for $U>\Delta$ each site is occupied by one electron with infinite spin degeneracy. Thus, for $t=0$ a transition occurs at a critical value $U_{c}=\Delta$. This transition is expected to persist for finite hopping amplitudes $t>0$.

A renewal of interest in the IHM started with the bosonization analysis of Fabrizio et al. (FGN) [12], where a two-transition scenario for the ground-state phase diagram of the 1D IHM was proposed. The key features of 
the FGN theory are the presence of an Ising-type transition from a CDW band-insulator phase at $U<U_{c h}^{c}$ into a BOW phase at $U_{c h}^{c}<U<U_{s p}^{c}$, and a continuous Kosterlitz-Thouless like transition into a MI phase at $U>U_{s p}^{c}$. In this scenario the charge gap vanishes only at $U=U_{c h}^{c}$ and the system might be "metallic" at this point. The second transition at $U=U_{s p}^{c}$ is connected with the closing of the spin gap, which is finite for all $U<U_{s p}^{c}$ and vanishes for $U>U_{s p}^{c}$. Thus, the bosonization phase diagram essentially supports the "exclusion principle" of the ground states.

Later on various attempts based on numerical tools have been performed to verify the FGN phase diagram for the half-filled IHM. In particular, exact diagonalization [13,18], valence bond techniques [15], quantum Monte Carlo [14], and DMRG [16,17] was used. Unfortunately, conflicting results have so far been reported in these studies regarding the nature of the transition and the insulating phases, the possibility of two rather than one critical point, or the appearance of BOW order.

Given the numerous unresolved issues we reinvestigate the ground-state properties of the IHM using the exact diagonalization Lanczos technique and the DMRG method. We verify the presence of at least one transition at a critical coupling $U_{c}(\Delta)$ from a band-insulator (BI) to a correlated insulator (CI) phase. On finite systems the transition originates from a ground-state level crossing with a change of the site-parity eigenvalue, which implies the vanishing of the optical excitation gap at $U_{c}$; Our DMRG results show that the spin and charge gaps remain nevertheless finite and equal at the transition. Above $U_{c}$ the charge and spin gaps split, the charge gap increases, while the spin gap decreases and we identify long-range BOW order with a spontaneous site-inversion symmetry breaking. The existence of a second transition is not unambiguously resolved within the accuracy of our DMRG data. Yet, the scaling of the BOW order parameter changes qualitatively with increasing $U$ indicative for a possible second smooth transition point where the spin gap closes.

We show that at $U<U_{c}(\Delta)$ the CDW-band insulator phase is realized. In this phase BOW and spin density wave (SDW) correlations are strongly suppressed, and the spin and charge gaps are equal and finite. The characteristic feature of the ground-state phases for $U>U_{c}(\Delta)$ is the coexistence of long range CDW order with either a long range $\mathrm{BOW}$ or algebraically decaying $\mathrm{BOW}$ and SDW correlations.

\section{SYMMETRY ANALYSIS}

A good starting point for understanding the existence of a phase transition in the IHM is to study the symmetry of the model manifestly seen in the limiting cases $U \ll$ $\Delta, t$ and $U \gg \Delta, t$. The IHM is invariant with respect to inversion at a site and translation by two lattice sites.
If we denote the site inversion operator by $P$, defined through

$$
P c_{i \sigma}^{\dagger} P^{\dagger}=c_{L-i \sigma}^{\dagger} \quad \text { for } \mathrm{i}=0, \cdots, \mathrm{L}-1,
$$

and $\hat{T}_{j}$ for a translation by $j$ sites, then any nondegenerate eigenstate $\left|\psi_{n}\right\rangle$ of $H$ must obey $P\left|\psi_{n}\right\rangle= \pm\left|\psi_{n}\right\rangle$ and $\hat{T}_{2}\left|\psi_{n}\right\rangle=\left|\psi_{n}\right\rangle$. Because $\left[H, \hat{T}_{1}\right] \neq 0$, any non-degenerate eigenstate $\left|\psi_{n}\right\rangle$ of $H$ is not an eigenstate of $\hat{T}_{1}$.

For the half-filled Hubbard model $(\Delta=\delta=0)$ the ground state has $P=+1$ only for $U=0$, and $P=-1$ for any $U>0$ [13]. However, in the IHM the phase transition from a renormalized BI to a CI occurs at some finite $U_{c}>0$. This suggests that the parity of the ground state remains even not only for $U=0$, but for all $U<U_{c}$. At $U_{c}$, a ground-state level crossing occurs on finite chains, as confirmed by exact diagonalization studies (see below), connected with a site-parity change.

For $U=0$ the ground state at half-filling is a CDW-BI. The alternating potential defines two sublattices, doubling the unit cell and opening up a band gap $\Delta$ for $U=0$ at $k= \pm \pi / 2$. The elementary spectrum consists of particle-hole excitations over the band gap. The charge $\left(\Delta_{C}\right)$ and spin $\left(\Delta_{S}\right)$ excitation gaps are equal: $\Delta_{C}=\Delta_{S}=\Delta$. We consider a system to be a BI when the criterion $\Delta_{S}=\Delta_{C}$ holds, where the spin and the charge gaps are given by

$$
\begin{aligned}
\Delta_{S} & =E_{0}\left(N=L, S_{z}=1\right) \\
& -E_{0}\left(N=L, S_{z}=0\right), \\
\Delta_{C} & =E_{0}\left(N=L+1, S_{z}=1 / 2\right) \\
& +E_{0}\left(N=L-1, S_{z}=1 / 2\right) \\
& -2 E_{0}\left(N=L, S_{z}=0\right),
\end{aligned}
$$

respectively. $E_{0}\left(N, S_{z}\right)$ is the ground-state energy, $L$ the system length, $N$ the number of electrons, and $S_{z}$ the $z$-component of the total spin. As we show below the BI phase is realized in the ground state of the IHM at $U<U_{c}$.

In the strong-coupling limit $U \gg \Delta, t$, the low-energy physics of the IHM is described by the following effective Heisenberg spin model [10]

$$
H_{e f f}=J \sum_{i} \mathbf{S}_{i} \cdot \mathbf{S}_{i+1}+J^{\prime} \sum_{i} \mathbf{S}_{i} \cdot \mathbf{S}_{i+2} .
$$

In Eq. (4) the exchange couplings are given by

$$
\begin{aligned}
J & =\frac{4 t^{2}}{U}\left[\frac{1}{1-x^{2}}-\frac{4 t^{2}}{U^{2}} \frac{\left.1+4 x^{2}-x^{4}\right)}{\left(1-x^{2}\right)^{3}}\right], \\
J^{\prime} & =\frac{4 t^{4}}{U^{3}} \frac{\left(1+4 x^{2}-x^{4}\right)}{\left(1-x^{2}\right)^{3}},
\end{aligned}
$$

where $x=\Delta / U$. This result (4) implies that in the strong-coupling limit of the IHM the low-energy physics is qualitatively similar to that of the Hubbard model, with modified exchange coupling constants $J$ and $J^{\prime}$. For 
next-nearest neighbor couplings $J^{\prime}<0.24 J$ the spin gap vanishes [25]. The coupling constants (5) satisfy the condition $J^{\prime}<0.24 J$ at least for $U>3.6 t$ for $\Delta \leq t$ and $U>3.6 \Delta$ for $\Delta>t$.

The effective spin model (4) is invariant with respect to translations by one lattice spacing, whereas the original IHM is invariant only with respect to translations by two lattice spacings. However, the doubling of the unit cell is ensured due to the charge degrees of freedom. For the standard Hubbard model at arbitrary $U \neq 0$ the number of doubly occupied sites $D$ in the ground state is finite. The exact Bethe-ansatz solution tells that $D$ scales as $(t / U)^{2}$ in the strong coupling limit and is given by [26]

$$
D=\sum_{i}\left\langle n_{i \uparrow} n_{i \downarrow}\right\rangle \simeq N A(t / U)^{2}\left[1+\mathcal{O}\left((t / U)^{2}\right)\right]
$$

where $A=4 \ln 2$. Contrary to the Hubbard model, where doublons are equally distributed on all sites of the system, the non-equivalence of sites in the IHM leads to different probabilities for finding a doublon on even or odd sites. Since doublons are spin singlets, their distribution is not influenced by the spin fluctuations. Since the energy of doublons on even and odd sites differ in $\Delta$ and assuming the scaling for the density of doublons as in Eq. (6), one easily obtains for the amplitude of the ionicity induced CDW in the strong coupling limit

$$
\begin{array}{r}
\frac{1}{N}\left(D_{\text {odd }}-D_{\text {even }}\right) \simeq A_{1} \frac{t^{2}}{U^{2}}\left[\frac{1}{(1-x)^{2}}-\frac{1}{(1+x)^{2}}\right] \\
=4 A_{1} \frac{t^{2}}{U^{2}} \frac{x}{\left(1-x^{2}\right)^{2}}\left[1+\mathcal{O}\left((t / U)^{2}\right)\right]
\end{array}
$$

where $A_{1}$ is a constant of order unity. Thus, although the effective spin Hamiltonian has a higher symmetry than the original model from which it was derived, the translational symmetry of the IHM is recovered due to the long range CDW pattern arising from the staggered doublon and holon distribution.

\section{EXACT DIAGONALIZATION RESULTS}

In order to explore the nature of the spectrum and the phase transition, we have diagonalized numerically small systems by the Lanczos method [27] similarly to earlier exact diagonalization calculations [11,13]. The energies of the few lowest eigenstates were obtained for finite chains with $L=4 n$ and periodic boundary conditions or $L=4 n+2$ with antiperiodic boundary conditions.

We first analyze short chains; for chain lengths $L \leq 16$ finite-size effects do not change the qualitative behavior discussed below. In Fig. 1, the lowest eigenenergies of the IHM for $\Delta=0.5 t, L=8$ and periodic boundary condition are shown as a function of $U$. At $U=1.3 t$, a

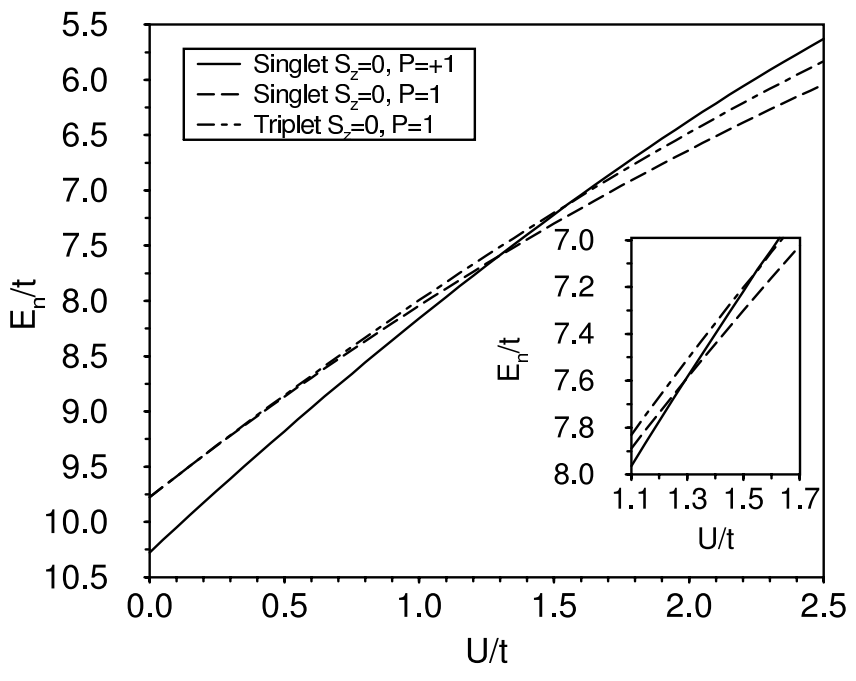

FIG. 1. Lowest energy eigenvalues of the IHM at half-filling for $L=8$ sites, periodic boundary conditions and $\Delta=0.5 t$.

level crossing of the two lowest eigenstates occurs. A nondegenerate eigenstate of the IHM has a well defined siteparity, so a ground-state level-crossing transition necessarily corresponds to a change of the site-parity eigenvalue.

For $U=0$, the IHM is easily diagonalized in momentum space by introducing fermionic creation operators $\gamma_{k \sigma b}^{\dagger}$ with a band index $b=1,2$ for the lower and upper bands, respectively, with the dispersion $E_{1 / 2}(k)=$ $\pm \sqrt{4 \cos ^{2}(k)+(\Delta / 4)^{2}}$ for momenta $-\pi / 2<k \leq \pi / 2$. For $U=0$ the first two degenerate excited states at half-filling always have negative site parity, because the ground state has $P=+1$, and the operator $\gamma_{q \sigma 2}^{\dagger} \gamma_{q \sigma 1}$ with $q=\pi / 2$ obeys

$$
P \gamma_{q \sigma 2}^{\dagger} \gamma_{q \sigma 1}=-\gamma_{q \sigma 2}^{\dagger} \gamma_{q \sigma 1} P
$$

The first two excited states shown in Fig. 1 are the spin singlet $\left(S=0, S_{z}=0\right)$ and triplet excitations $(S=1$, $\left.S_{z}=0\right)$, created from the ground state by applying the operators

$$
\begin{aligned}
& \frac{1}{\sqrt{2}}\left(\gamma_{q \uparrow 2}^{\dagger} \gamma_{q \uparrow 1}-\gamma_{q \downarrow 2}^{\dagger} \gamma_{q \downarrow 1}\right), \\
& \frac{1}{\sqrt{2}}\left(\gamma_{q \uparrow 2}^{\dagger} \gamma_{q \uparrow 1}+\gamma_{q \downarrow 2}^{\dagger} \gamma_{q \downarrow 1}\right),
\end{aligned}
$$

respectively. Thus both excited states have total momentum $k_{\text {tot }}=0$ and negative site parity. For $U>0$, these degenerate excited states split in energy. Exact diagonalization of finite IHM rings therefore identifies one critical $U_{c}>0$, separating a BI with $P=+1$ at $U<U_{c}$ from a CI with $P=-1$ for $U>U_{c}$. 


\section{DMRG RESULTS}

\section{A. Excitation gaps}

In order to access the transition scenario in the long chain-length limit, we have studied chains up to $\mathrm{L}=512$ using the DMRG method [28-30]. The fact that the transition at $U_{c}$ is connected to a change in inversion symmetry requires some caution when open boundary conditions (OBC) are used in DMRG studies. For OBC and $L=2 n$ the IHM is not reflection symmetric at any site. Thus, the ground state does not have a well defined site parity, and the level-crossing transition is absent. To overcome this problem, one might try to use chains with OBC and an odd number of sites $L=2 n+1$, since the Hamiltonian in this case is reflection symmetric with respect to the site $i_{c}$ in the center of the chain, and a site inversion operator is well defined by

$$
P c_{i_{c} \sigma}^{\dagger} P^{\dagger}=c_{L-1-i_{c} \sigma}^{\dagger} \quad i=0, \ldots, L-1 .
$$

To test whether this is an improved choice we have calculated the site parity of the ground state for $U=0$ analytically for different chain lengths $L=2 n+1$ and found

$$
P\left|\psi_{0}\right\rangle=(-1)^{n}\left|\psi_{0}\right\rangle
$$

On the other hand, if one extends the idea of Gidopoulos et al. [13] for the determination of the site parity to chains with $L=2 n+1$ for $U \gg t$, one obtains

$$
P\left|\psi_{0}\right\rangle=(-1)^{\left[\sum_{m=1}^{L-1} m\right]}\left|\psi_{0}\right\rangle=(-1)^{n}\left|\psi_{0}\right\rangle \text {. }
$$

Thus, the parity eigenvalue of the ground state is the same at $U=0$ and $U \gg t$ for a given chain length, and no level crossing occurs. Due to the fact that the sharp transition at a well defined $U_{c}$ does not exist in the finite-chain results for $\mathrm{OBC}$, the extrapolation is a rather subtle problem, since a sharp transition feature has to be identified from the extrapolation of smooth curves. This requires the use of quite long chains in the critical region.

In Fig. 2 extrapolated results are shown for the spin and charge gaps $\Delta_{S}$ and $\Delta_{C}$, respectively. Calculations were performed with OBC for chains of lengths $L=\{30,40,50,60\}$, and additionally up to $L=512$ in the transition region around the estimated $U_{c}$. We assume a scaling behavior of $\Delta_{C}$ and $\Delta_{S}$ of the form [32]

$$
\Delta_{i}(L)=\Delta_{i}^{\infty}+\frac{A_{i}}{L}+\frac{B_{i}}{L^{2}},
$$

where $i \in\{S, C\}$. The extrapolation for $L \rightarrow \infty$ is then performed by fitting this polynomial in $1 / L$ to the calculated finite-chain results. We note that different finite-size scaling formulas were proposed in the literature mainly when periodic or antiperiodic boundary conditions were used [13].

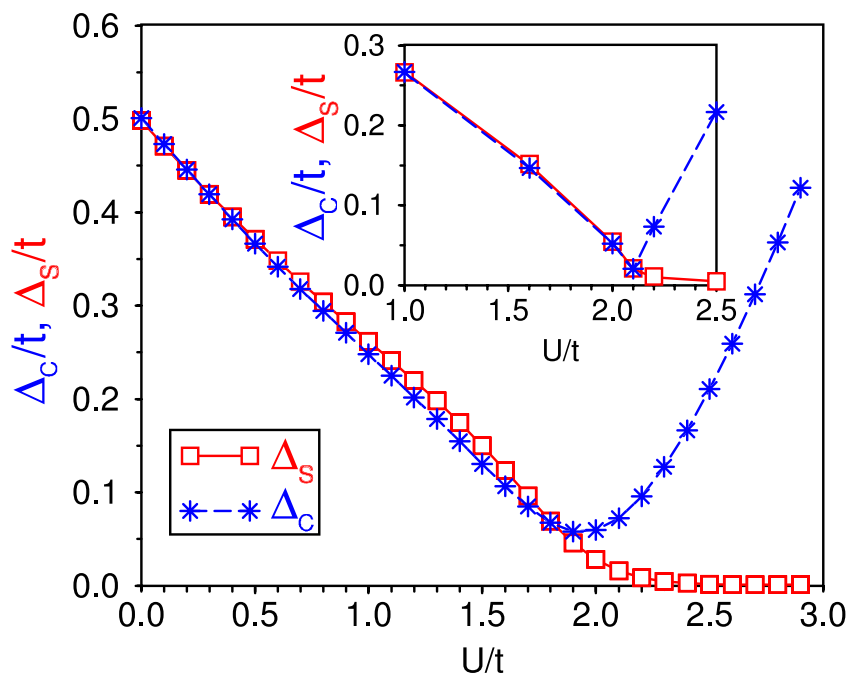

FIG. 2. Results for the spin $\left(\Delta_{S}\right)$ and charge $\left(\Delta_{C}\right)$ gaps of the IHM at half-filling with $\Delta=0.5 t$ as a function of $U$. Energies were obtained by DMRG calculations on open chains with $L=\{30,40,50,60\}$ (main plot) and up to $L=512$ (inset), and extrapolated to the limit of infinite chain length.

As can be seen from the main plot in Fig. 2, extrapolating the results for $L=\{30,40,50,60\}$ does indeed not give a sharp transition behavior. As illustrated in the inset, adding results for $L$ up to 512 in the critical region changes the picture considerably. Within numerical accuracy the charge and spin gaps remain equal and finite up to a critical $U_{c} \approx 2.1 t$, where a sharp kink for $\Delta_{C}$ is observed. Importantly, $\Delta_{C}$ does not close at the critical point. We emphasize that the magnitude of $\Delta_{C}$ and $\Delta_{S}$ at the transition point is sufficiently larger than our numerical uncertainty in the finite size scaling analysis and therefore allows for a safe conclusion. $\Delta_{C}=\Delta_{S}>0$ at the transition is in fact not in conflict with an underlying ground-state level crossing. If the ground states of the different site-parity sectors become degenerate, the only rigorous consequence is the closing of the optical excitation gap. The selection rules for optical excitations allow only for transitions between states of different siteparity. Furthermore, optical transitions occur within the same particle number sector. The optical gap is therefore by definition distinct from the charge gap Eq. (2) which involves the removal or the addition of a particle. The critical point $U_{c}$ of the IHM has the remarkable peculiarity that the optical gap closes while $\Delta_{C}$ remains finite. Above $U_{c}$ the charge and spin gaps split indicating that the corresponding insulating phase is no longer a BI. $\Delta_{S}$ continuously decreases with increasing $U$ and becomes unresolvably small above $U \sim 2.5 t$ within the achievable numerical accuracy.

The result, that $\Delta_{C}$ and $\Delta_{S}$ remain finite and equal at the transition is in agreement with the data obtained by Qin et al. [17]. These authors performed DMRG calculations for the IHM with $\Delta=0.6 t$ for open chains up to 
$L=600$ sites. They observed a surprising non-monotonic scaling behavior of $\Delta_{S}$ with $L$ for values of $U$ close to the critical $U_{c}$, i.e. for chain lengths $L>300 \Delta_{S}$ started to increase again. It remains unclear whether this is due to loss of DMRG accuracy with increasing chain lengths and keeping a fixed number of states in the DMRG algorithm. In contrast, our data always show a monotonous scaling with $1 / L$. DMRG calculations for the IHM with $\Delta=t$ have also been performed by Takada and Kido for chains up to $L=400$ sites [16]. The authors interpret their results in the region close to $U_{c}$ in favour of a two-transition scenario similar to that of FGN [12]. However, as we will show below the accuracy of the currently available DMRG data is not enough to provide a stringent argument in favour of this interpretation.

For comparison we show in Fig. 3 the spin and charge gaps versus $U$ in the Peierls Hubbard model. As we observe this model is distinctly different from the IHM. This is also a band-insulator at $U=0$, but in contrast to the IHM has $\Delta_{C}>\Delta_{S}>0$ for any value $U>0$, i.e. the phase transition from the Peierls band-insulator to the correlated insulator occurs at $U_{c}=0$. So although the Peierls and the ionic BI for $U=0$ similarly possess an excitation gap at the Brillouin zone boundary, applying a Coulomb $U$ leads to distinctly different behavior in both cases. The origin of the different behaviors must be traced to the fact that the Hubbard interaction and the ionic potential compete locally on each site, while the Peierls modulation of the hopping amplitude tends to move charge to the bonds between sites, thereby avoiding conflict with the Hubbard term.

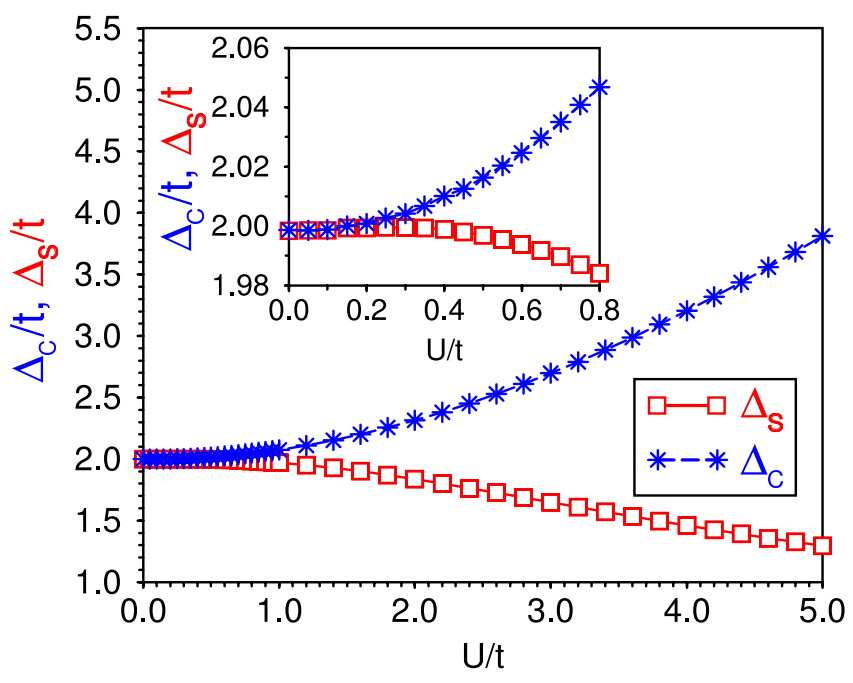

FIG. 3. Results for the spin $\left(\Delta_{S}\right)$ and charge $\left(\Delta_{C}\right)$ gaps vs. $U$ of the Peierls-Hubbard model at half-filling with a modulation of the hopping amplitude $\delta=0.5$. Energies were obtained by DMRG calculations on open chains with $L=\{30,40,50,60\}$.

The spin-Peierls physics of the Peierls Hubbard model at large $U$ evolves smoothly with decreasing $U$ into the physics of a spin-gapped CI-BOW state in the weak- coupling limit, which is characterized by long-range staggered bond-density correlations

$$
\begin{aligned}
g_{b}(r) & =\frac{1}{L} \sum_{i}\left\langle\psi_{0}|b(i) b(i+r)| \psi_{0}\right\rangle, \\
b(i) & =\sum_{\sigma}\left(c_{i \sigma}^{\dagger} c_{i+1 \sigma}+H . c .\right) .
\end{aligned}
$$

For $U \gg t$ the low-energy physics of the Peierls Hubbard model is described by the spin-Peierls Heisenberg Hamiltonian with a staggered exchange interaction and a dimerization induced spin gap [31].

\section{B. Correlation functions}

The important question remains about the nature of the insulating phase of the IHM for $U>U_{c}$. To further analyze the $\mathrm{BI}$ and $\mathrm{CI}$ phases below and above $U_{c}$, we have evaluated site- and bond-charge distribution functions as well as spin-spin correlation functions. In

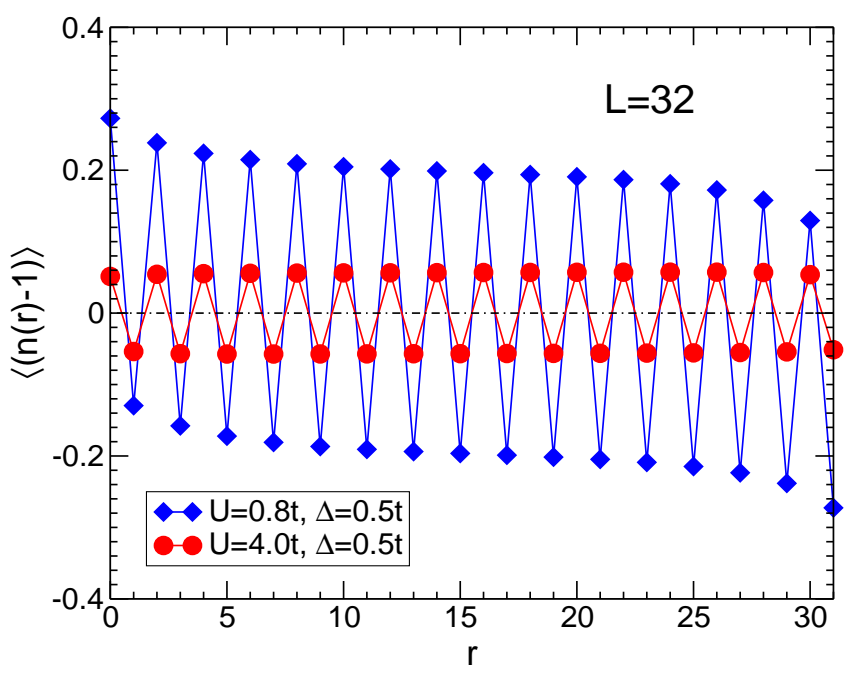

FIG. 4. Electron density distribution in the ground state of the IHM for $\Delta=0.5 t$ and $U=0.8 t$ (diamonds) and $U=4 t$ (circles). Results were obtained by DMRG calculations on an open $L=32$ chain.

Fig. 4 we show the charge distribution $\langle 0|(n(r)-1)| 0\rangle$ $\left(n(r)=n_{r, \uparrow}+n_{r, \downarrow}\right)$ in the ground state $|0\rangle$ for the IHM at $U=0.8 t<U_{c}$ and $U=4 t>U_{c}$ for a $L=32$ chain. The alternating pattern in the density distribution is well pronounced not only in the BI but also in the CI phase far beyond the critical point at $U \gg U_{c}$. For the $L=32$ chain the CDW is well established at distances $l \sim L / 2$ even at $U=4 t$ and its amplitude remains almost unchanged in the finite-size scaling analysis (see upper inset in Fig. 5). The main plot in Fig. 5 shows that the staggered component of the charge density decreases smoothly with increasing $U$. Close above the transition point near $U=2 t$ one observes an anomaly, i.e. a slight decrease of the 


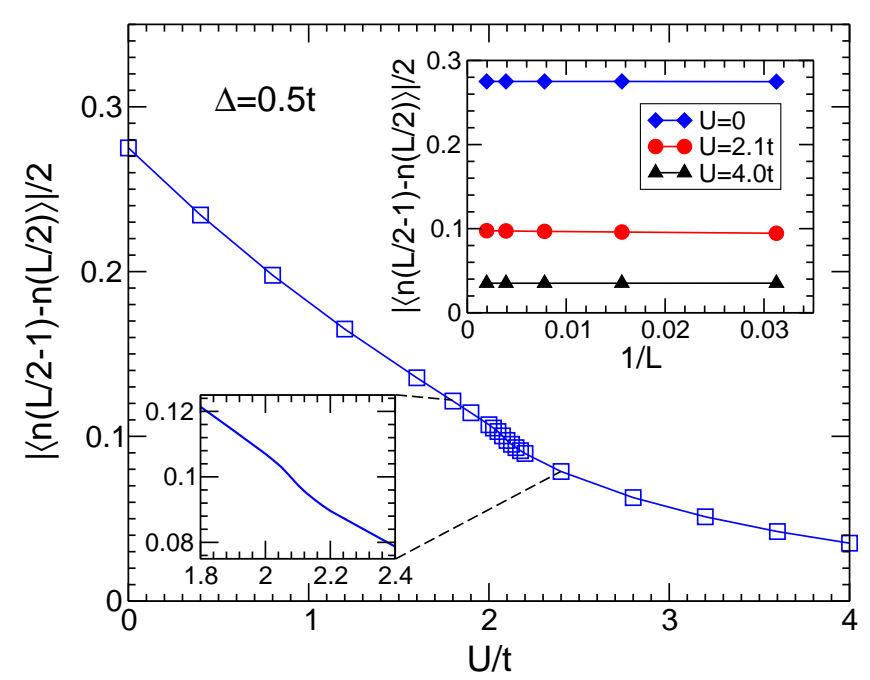

FIG. 5. Staggered charge density component vs. $U$, for $\Delta=0.5 t$ and $L=512$ (main plot). Its scaling behavior, for $U=0$ (diamonds), $U=2.1 t$ (circles) and $U=4.0 t$ (triangles) is shown in the inset.

CDW amplitude accompanied by a change in curvature; this anomaly can be clearly identified in the enlargement shown in the lower inset. The anomaly will find a natural explanation in the discussion below. Our numerical data show that the alternating pattern in the electron density distribution in the IHM remains for arbitrary finite $U$. Thus, the ionic potential enforces long range CDW order for all interaction strengths.

Fig. 6 shows the DMRG results for the spin-spin correlation function $\left\langle 0\left|S^{z}(L / 2) S^{z}(L / 2+r)\right| 0\right\rangle$ for the IHM at $U=0.8 t<U_{c}$ and $U=4 t>U_{c}$ in comparison with the Hubbard chain. In the BI phase at $U=0.8 t$ the SDW correlations are quickly suppressed after a few lattice spacings. At $U=4 t$ the amplitude of the SDW correlations in the CI phase of the IHM is slightly reduced in comparison to the Hubbard model at the same value of $U$. However, the large distance behaviors of the spin correlations in the CI phase of the IHM and the MI phase of the Hubbard model are quite similar and become almost indistinguishable (see the data in the inset for $U=4 t$ ). On the other hand, the long distance behavior of the spin-spin correlation function at $U=2.1 t$ and $\Delta=0.5 t$, i.e. close above the transition, manifestly supports the finiteness of the spin gap (see inset). This may be viewed as an indication for existance of two different phases above $U_{c}$. But we cautiously point out that it is hard to judge on the persistence or vanishing of $\Delta_{S}$ far above $U_{c}$ in the CI phase from the finite chain spin correlators alone.

To address the BOW ordering tendencies in the CI phase we have calculated the ground-state distribution of the bond-charge density Eq. (15). Fig. 7 shows the results of the DMRG calculations for the $L=32$ IHM and the Hubbard chain at $U=0.8 t$ and $U=2.6 t$. The boundary effect for an open chain is strong and leads to

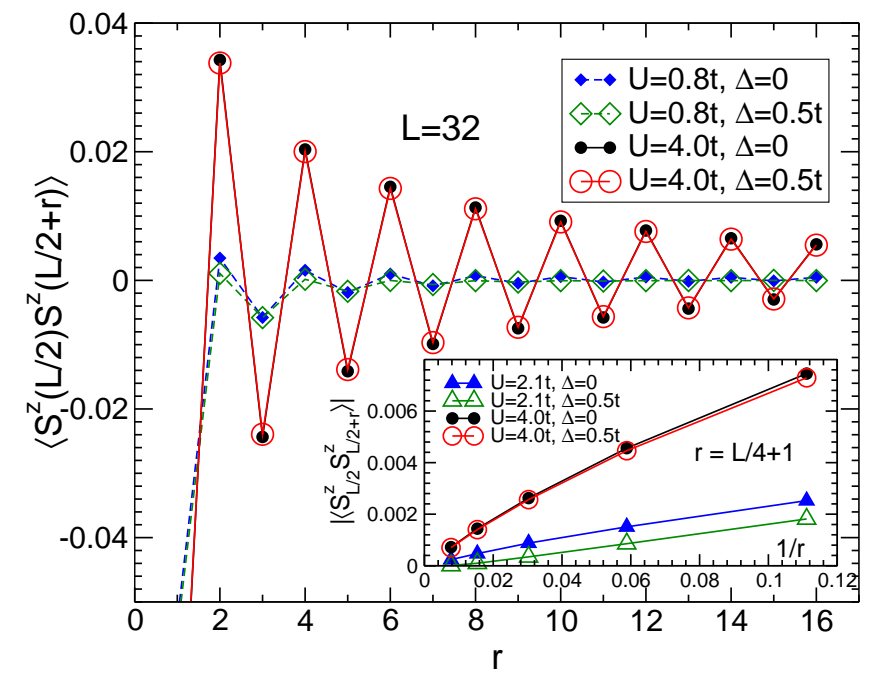

FIG. 6. Spin-spin correlation function in the ground state of the IHM for $\Delta=0.5 t$ (open symbols) and the Hubbard model $(\Delta=0)$ (full symbols) at $U=0.8 t$ (diamonds) and $U=4 t$ (circles). Chain length $L=32$ (main plot). Inset: Scaling of the spin-spin correlation function at $r=L / 4+1$ for $U=2.1 t$ (up and down triangles) and $U=4 t$ (circles) for the IHM (open symbols) and the Hubbard model (full symbols)

a modulation of the bond density already for the pure Hubbard model. Interestingly, the same behavior was also observed previously for the bond expectation value $\left\langle\mathbf{S}_{i} \cdot \mathbf{S}_{i+1}\right\rangle$ in open antiferromagnetic Heisenberg spin$1 / 2$ chains [29]. The detailed comparison with the Hubbard chain in Fig. 7 reveals, that the ionic potential leads to a reduction of the bond-density oscillations at $U<U_{c}$, while in the CI phase at $U>U_{c}$ their amplitude slightly increases. The enhancement of BOW correlations above $U_{c}$ must simultaneously weaken the CDW amplitude. This naturally explains the slight downward curvature near $U_{c}$ in the staggered charge density component shown in Fig. 5.

In order to explore the possibility towards true long range BOW ordering in the IHM above $U_{c}$ we plot in Fig. 8 the staggered bond-density versus $U$ in the center of long, open chains with $L=256$ and $L=512$. In the BI phase this quantity is essentially zero. At the transition point the staggered component of the bond density increases rapidly, and on further increasing $U$ it starts to decrease smoothly. However, the staggered bond density remains finite for any $U>U_{c}$ on these long but finite chains and vanishes only in the limit $U \rightarrow \infty$.

Naturally it is necessary to perform a finite size scaling analysis for this quantity. Fig. 9 shows its chain length scaling behavior for $U=0, U=2.1 t$, and $U=4 t$. Two conclusions can be drawn from these results: In the absence of the interaction the staggered bond density clearly extrapolates to zero - as expected for a conventional BI. In the CI phase close above the transition the upward curvature of the staggered bond density vs. $1 / L$ 


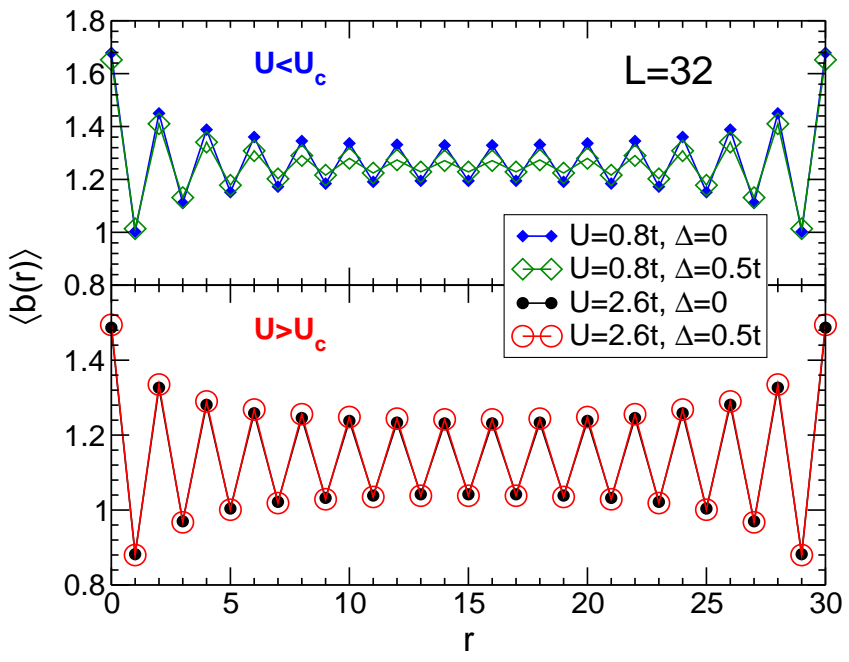

FIG. 7. Bond-charge density of the IHM for $\Delta=0.5 t$ (open symbols) and the Hubbard model (full symbols) at $U=0.8 t$ (diamonds) and $U=2.6 t$ (circles).

points to a finite value in the infinite chain length limit, i.e. long range BOW order. However, the scaling behavior changes in the CI phase far above the transition point. Interestingly, the scaling behavior in the IHM far above $U_{c}$ starts to reseamble the results for the pure Hubbard model, for which the staggered bond density has to vanish in the thermodynamic limit. The qualitative change in the scaling behavior of the staggered bond density may again be viewed indicative for a possible second phase transition. Summarizing the results for the SDW and BOW correlations we conclude that there is evidence for two phases for $U>U_{c}$. Close above $U_{c}$ long range BOW order develops in the ground state of IHM while far above the transition point the coorrelation functions become almost identical to those of the Hubbard model. Yet, long range CDW order exists for all $U$. A precise location of a second transition point is however not possible from the currently awailable DMRG data.

\section{CONCLUSIONS}

From the finite chain DMRG studies and finite size scaling analyses we draw the following conclusions for the ground-state phase diagram of the IHM: the ionic potential leads to long range CDW order for all interaction strengths. The data resolve one transition point from the BI to the CI phase. Remarkably, at the transition $\Delta_{C}=\Delta_{S}$ and both remain finite. Close above the transition, i.e. for $0<\left(U / U_{c}\right)-1 \ll 1$, we identify a clear signal for long range BOW order. With increasing $U$ above $U_{c}$ the finite size scaling behavior of the staggered bond density and spin-spin correlation function changes qualitatively and approaches the scaling behavior of the Hubbard model. With the current chain length and DMRG accuracy limitations it is not possible to precisely iden-

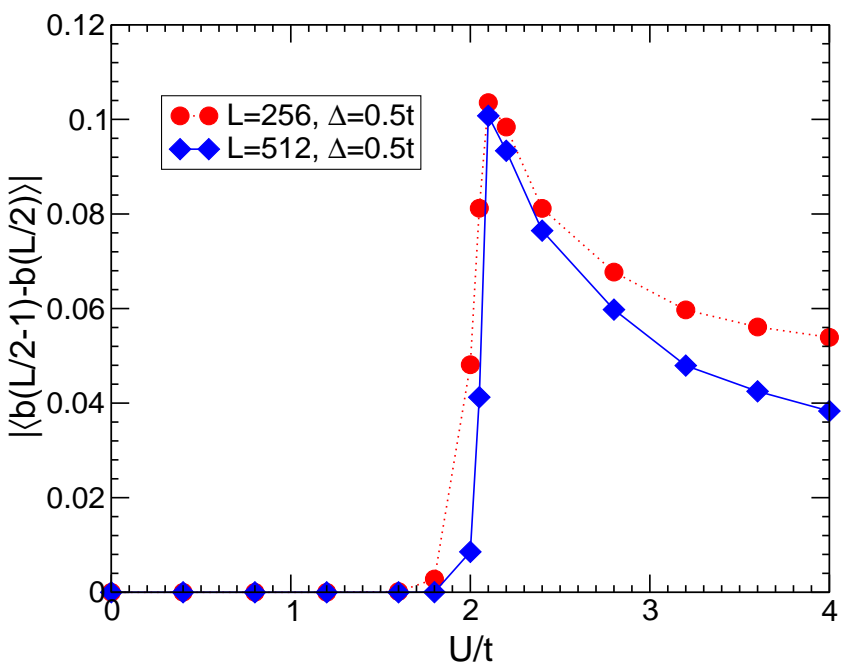

FIG. 8. Staggered bond-density component vs. $U$ near the center of the $L=256$ (circle, dotted line) and $L=512$ (diamonds, solid line) chain.

tify and locate the second transition point. The phase with BOW order necessarily has a finite spin excitation gap. If BOW seizes to exist above a second critical value of $\mathrm{U}$, the spin gap has to vanish simultaneously leading to identical large distance decays of SDW and BOW correlations.

The insulator-insulator transition at $U_{c}(\Delta)$ on finite periodic chains results from a ground-state level crossing of the two site-parity sectors. The optical excitation gap therefore has to vanish at $U_{c}$; remarkably, the DMRG data reveal that at the critical point $\Delta_{C}$ ans $\Delta_{S}$ remain both finite and equal. This itself clearly indicates the existanse of a CI phase with $\Delta_{C}>\Delta_{S}>0$ which originates from the appearance of long range staggered bond density order. The distinction between the optical and the charge gap is therefore of key importance for the structure of the insulating phases and the phase transitions of the IHM. The investigation of the optical conductivity in the critical region is therefore a demanding task for future work on the complex physics of the ionic Hubbard model.

\section{ACKNOWLEDGMENTS}

We thank R. Noack, B. Normand, H. Fehske, and T. Vekua for helpful discussions. This work was supported by the Deutsche Forschungsgemeinschaft (DFG) through SP 1073. APK also acknowledges support through Sonderforschungsbereich 484 of the DFG. GIJ and MS acknowledge support by the SCOPES grant N 7GEPJ62379. 


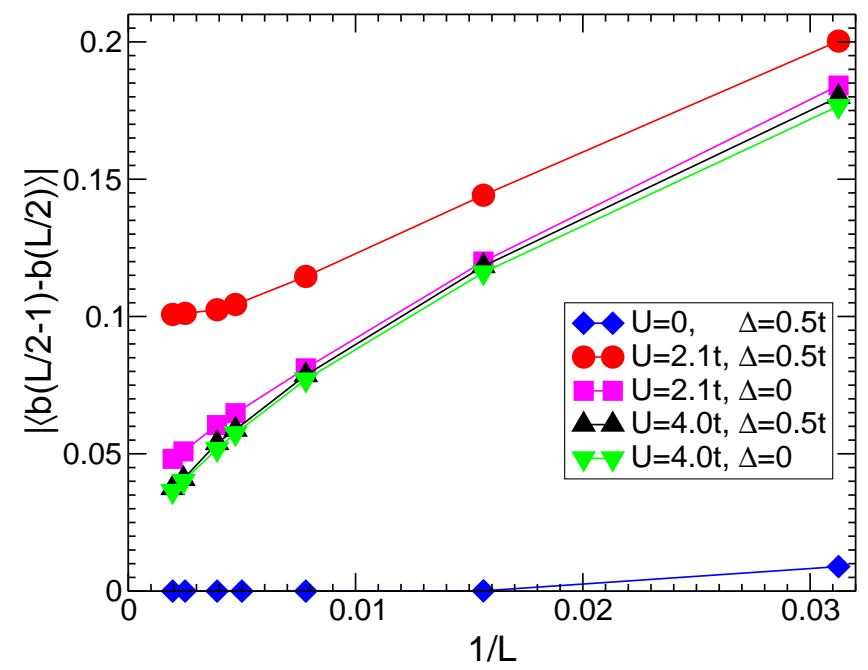

FIG. 9. Scaling behavior of the staggered bond-density component in the IHM near the center of the chain for $U=0$ (BI, diamonds), $U=2.1 t$ (CI close above the transition, circles), $U=4.0 t$ (CI far above the transition, up triangles) and in the Hubbard model for $U=2.1 t$ (squares) and $U=4.0 t$ (down triangles).

[1] For a review see: M. Imada, A. Fujimori, and Y. Tokura, Rev. Mod. Phys. 70, 1039 (1998).

[2] D. H. Lee and R. Shankar, Phys. Rev. Lett. 65, 1490 (1990).

[3] E. Lieb and F. Y. Wu, Phys. Rev. Lett. 20, 1445 (1968)

[4] D. C. Mattis, The Theory of Magnetism, vols. I and II, New York Springer-Verlag (1987)

[5] V. J. Emery, in Highly Conducting One-Dimensional Solids, Edited by J.T. Devreese, E. P. Evrard and V.E. Van Doren (New York, Plenum) (1979) p. 247

[6] M. Nakamura, J. Phys. Soc. Jpn. 68, 3123 (1999); Phys. Rev. B 61, 16377 (2000).

[7] P. Sengupta, A.W. Sandvik, and D.K. Campbell, Phys. Rev. B 65, 155113 (2002).

[8] H. Fehske, A. P. Kampf, M. Sekania, G. Wellein Eur. Phys. J. B 31 , 11 (2003)

[9] G. I. Japaridze, Phys. Lett. A 201, 239 (1995) G. I. Japaridze and A. P. Kampf, Phys. Rev. B 59, 12822 (1998)

[10] N. Nagaosa and J. Takimoto, J. Phys. Soc. Jpn. 55, 2735 (1986); N. Nagaosa, ibid. 55, 2754 (1986).

[11] R. Resta and S. Sorella, Phys. Rev. Lett. 74, 4738 (1995).

[12] M. Fabrizio, A. O. Gogolin, and A. A. Nersesyan, Phys. Rev. Lett. 83, 2014 (1999); Nucl. Phys. B 580, 647 (2000).

[13] N. Gidopoulos, S. Sorella, and E. Tosatti, Eur. Phys. J. B 14, 217 (2000).

[14] T. Wilkens and R. M. Martin, Phys. Rev. B 63, 235108 (2001).

[15] Y. Anusooya-Pati, Z. G. Soos, and A. Painelli, Phys. Rev. B 63, 205118 (2001).

[16] Y. Takada and M. Kido, J. Phys. Soc. Jpn. 70, 21 (2001).

[17] S. Qin, J. Lou, T. Xiang, G. Tian, and Z. Su, preprint cond-mat/0004162, unpublished.
[18] M.E. Torio, A.A. Aligia, and H.A. Ceccatto, Phys. Rev. B 64, 121105 (2001).

[19] J. B. Torrance et al., Phys. Rev. Lett. 46, 253 (1981); ibid. 47, 1747 (1981).

[20] T. Mitani et al., Phys. Rev. Lett. 53, 842 (1984); Y. Tokura et al., Sol. State Comm. 43, 757 (1982); Mol. Cryst. Liq. Cryst. 125, 71 (1985); T. Mitani et al., Phys. Rev. B 35, 427 (1987); K. Takaoka et al., ibid. 36, 3884 (1987); Y. Tokura et al., ibid. 38, 2215 (1988).

[21] T. Egami, S. Ishihara, and M. Tachiki, Science 261, 1307 (1993).

[22] S. Ishihara, T. Egami, and M. Tachiki, Phys. Rev. B 49, 8944 (1994).

[23] T. Neumann et al., Phys. Rev. B 46, 10623 (1992).

[24] H.M. McConnell et al., Annu. Rev. Phys. Chem. 17, 237 (1966).

[25] F. D. M. Haldane, Phys. Rev. B 25, 4925 (1982); K. Okamoto and K. Nomura, Phys. Lett. A 169, 433 (1993); G. Castilla, S. Chakravarty, and V. J. Emery, Phys. Rev. Lett. 75, 1823 (1995).

[26] J. Carmelo and D. Baeriswyl, Phys. Rev. B 37, 7541 (1988)

[27] C. Lanczos, J. Res. Natl. Bur. Stand. 45, 255 (1950).

[28] S. R. White, Phys. Rev. Lett. 69, 2863 (1992); Phys. Rev. B 48, 10345 (1993).

[29] S. R. White, Phys. Rev. B 48, 10345 (1993).

[30] I. Peschel, X. Wang, M. Kaulke, K. Hallberg (Eds.): Density-Matrix Renormalization, Springer (1999).

[31] L. N. Bulaevskii, A. I. Buzdin, and D. I. Khomskii, Solid State Commun. 27, 5 (1978); M. C. Cross and D. S. Fisher, Phys. Rev. B 19, 402 (1979).

[32] R. Noack, 2002 private communication. 